ARTICLE OPEN

Check for updates

\title{
Cardiovascular deconditioning during long-term spaceflight through multiscale modeling
}

\author{
Caterina Gallo (iD), Luca Ridolfi ${ }^{1}$ and Stefania Scarsoglio (iD ${ }^{1 凶}$
}

Human spaceflight has been fascinating man for centuries, representing the intangible need to explore the unknown, challenge new frontiers, advance technology, and push scientific boundaries further. A key area of importance is cardiovascular deconditioning, that is, the collection of hemodynamic changes-from blood volume shift and reduction to altered cardiac function-induced by sustained presence in microgravity. A thorough grasp of the $0 \mathrm{G}$ adjustment point per se is important from a physiological viewpoint and fundamental for astronauts' safety and physical capability on long spaceflights. However, hemodynamic details of cardiovascular deconditioning are incomplete, inconsistent, and poorly measured to date; thus a computational approach can be quite valuable. We present a validated 1D-0D multiscale model to study the cardiovascular response to long-term $0 \mathrm{G}$ spaceflight in comparison to the $1 \mathrm{G}$ supine reference condition. Cardiac work, oxygen consumption, and contractility indexes, as well as central mean and pulse pressures were reduced, augmenting the cardiac deconditioning scenario. Exercise tolerance of a spaceflight traveler was found to be comparable to an untrained person with a sedentary lifestyle. At the capillary-venous level significant waveform alterations were observed which can modify the regular perfusion and average nutrient supply at the cellular level. The present study suggests special attention should be paid to future long spaceflights which demand prompt physical capacity at the time of restoration of partial gravity (e.g., Moon/Mars landing). Since spaceflight deconditioning has features similar to accelerated aging understanding deconditioning mechanisms in microgravity are also relevant to the understanding of aging physiology on the Earth.

npj Microgravity (2020)6:27; https://doi.org/10.1038/s41526-020-00117-5

\section{INTRODUCTION}

The cardiovascular system undergoes a constellation of important hemodynamic changes in microgravity-such as fluid shift of about 2 I from the legs to upper body, total blood volume reduction of around $11 \%$, decrease in cardiac muscle mass of over $10 \%$, and reduced exercise capability of up to $20 \%$ in the first 30 days-which lead to cardiovascular deconditioning ${ }^{1-8}$. Understanding $0 \mathrm{G}$ configuration in detail is particularly crucial to prevent and mitigate disabling effects through targeted countermeasures, and thus ensure the full health and well-being of astronauts during spaceflight missions ${ }^{2}$. A better comprehension of deconditioning mechanisms can answer open questions on readaptation for spaceflight beyond 1 year. It is currently unknown whether very lengthy missions lead to an amplification of reversible changes already known or the emergence of unrecognized and irreversible alterations in cardiovascular function ${ }^{3}$. Moreover, even if cardiovascular deconditioning is not extreme during spaceflight, it can later become hazardous at the time of reentry on the Earth or partial gravity restoration (e.g., Moon or Mars landing), when immediate demands for physical capacity are required and cannot be met. In view of future Moon or Mars missions, long-term cardiovascular deconditioning will need to be addressed to: (i) evaluate the efficacy of countermeasures and to optimize them in order to guarantee the astronauts' safety; and (ii) establish risk thresholds, especially for physical activity after landing. Additionally, as space science is an extraordinary investment multiplier (9:1 return on investment by way of spin-offs from space technology), understanding space deconditioning can have impacts on other clinical conditions and on aging research ${ }^{3}$.
Although the overall scenario of cardiovascular deconditioning is widely accepted, the extreme difficulty of performing clinical measurements, the unsystematic data collection on older missions, the limited number of human space missions, and the heterogeneity of the missions (in terms of reference baseline posture, crew, objectives, countermeasures, duration, etc.) make the details of cardiovascular responses controversial and restricted to a modest number of cardiac parameters ${ }^{9-12}$. To the best of our knowledge, hemodynamic parameters related to the right heart, oxygen consumption, and exercise tolerance are barely known in microgravity. Almost no information at the vascular level regarding organ blood supply and wave propagation mechanisms is available.

Ground-based analogs-such as bed rest studies and water immersion-are not fully representative of microgravity as they introduce artificial horizontal and hydrostatic pressure gradients respectively. Parabolic flight temporarily reproduces $0 \mathrm{G}$ effects with each session lasting only $20-30 \mathrm{~s}^{13}$.

Given the above difficulties, the computational approach is a recent and promising tool to study the human body's response to microgravity conditions ${ }^{14-18}$, as it can be used to investigate variables and processes which are hard to measure, as well as predict different scenarios and optimal countermeasures. In particular, reduced order modeling seems to be the most appropriate strategy, as it is a reasonable balance between the suitable level of local detail and the overall response (see, among the most recent ${ }^{19-23}$ ). Multiscale modeling has already provided useful insights into aging ${ }^{24,25}$, coronary pathologies ${ }^{26,27}$, and cardiac arrhythmias ${ }^{28-30}$. 
The present study aims to computationally shed light on the cardiovascular knowledge gaps previously described, following a comprehensive bibliographic investigation of more than 50 studies of cardiovascular deconditioning during microgravity. Among the available literature we only focused on the most recent long-term spaceflight data. This allowed us to extract information to characterize the steady-state cardiovascular condition for longterm (at least 5 months) spaceflight. In order to model the typical long-term response, we prioritized studies having only routine and customary countermeasures, and avoided spaceflights with specific/peculiar ad hoc countermeasures. The main cardiovascular features of the $0 \mathrm{G}$ adaptation point are: blood shift from lower to upper body, total blood volume reduction, reduced cardiac function and volume, increase of lower extremity venous compliance, arterial resistance variation, and baroreflex response alteration. Our goal is to quantitatively describe in detail the cardiovascular response through a comprehensive 0D-1D multiscale model, which has been successfully tested by heart pacing and open-loop response ${ }^{31}$. The OD-1D heart-arterial hemodynamics of the complete model has been previously validated to subject-specific and pathological conditions $s^{25,28,32-34}$. The physical-based model combines a 1D description of the arterial tree together with a lumped parameterization of the remaining regions, i.e., venous return, heart chambers, pulmonary circulation, and baroreceptor regulation (see the Methods for more detail and for the limitations of the present approach). In particular, we compared 1G supine and OG spaceflight conditions in order to: (i) understand the underlying mechanisms leading to cardiovascular deconditioning, and (ii) describe the hemodynamics of zones for which clinical data are not yet feasible and accurate. The adoption of the $1 \mathrm{G}$ supine condition as a reference baseline has a twofold justification: (i) supine is the reference medical position defining the benchmark for physiological cardiovascular behavior in clinical practice; (ii) over recent decades, cardiovascular modeling (see, among others, ${ }^{19-21,23,35}$ ) has been derived which simulates the posture of primary medical interest and has developed a robust, data-based, and well-accepted parameter setting, which serves as a solid reference point for parameter tuning. Changes occurring in OG condition described hereafter are always reported with respect to the (chosen) $1 \mathrm{G}$ supine baseline. The present study provides computational-based insights into several cardiac parameters and hemodynamic variables (in terms of time-series, average distribution throughout the body, and waveform alteration), which are currently unexplored.

\section{RESULTS}

Results are organized showing the main cardiac parameters first. Whenever possible, to validate the modeling response we compared our results to available literature data of long-term spaceflights which employed routine countermeasures and which referred to the $1 \mathrm{G}$ supine condition. The cardiovascular system was divided into four areas: cerebral, cardio-thoracic, abdominal, and lower limbs. Special focus was given to the proximal-to-distal pathway of the arterial tree. Time-series, characteristic levels (i.e., mean, maximum, excursion) and waveform changes of pressures $(P)$, flow rates $(Q)$, and volumes $(V)$, were then evaluated for representative sites of each cardiovascular region. In the following, supine $1 \mathrm{G}$ configuration on the Earth is depicted with blue curves, while long-term OG spaceflight is depicted with red curves. For each configuration results are referred to a generic steady-state heartbeat, so that $t \in[0, R R]$, where $R R[\mathrm{~s}]$ is the cardiac beating period and $H R=60 / R R$ [bpm] is the heart rate.

\section{Cardiac parameters}

The most important cardiac parameters are reported in Table 1 (see Supplementary Information for definitions). We first compared present outcomes with literature data. To this end, it should be noted that the detailed cardiovascular response in literature is quite discordant and debated. As comprehensively reported by Norsk $^{12}$, for different hemodynamic parameters-such as heart rate, arterial pressure, cardiac output, stroke volume-there are conflicting results, which depend on the level of adopted countermeasures, the mission duration, and, most importantly, the baseline (upright/sitting/supine) reference $1 G$ condition. In view of the above considerations very few assessed metrics were available for full comparison and, among these, we selected the most recent studies involving long-term spaceflights (around 5 months). Thus, collected measurements are the most reliable results which we adopted as reference values for the present study.

A first validation comes from the left ventricular contractility parameters: they all reduced $\left(-18.32 \%\right.$ for $S V,-9.30 \%$ for $V_{\text {lved }}$ $-9.93 \%$ for $E F$ ) except for a slight increase of $V_{\text {lves, }}+4.75 \%$, and are in good agreement with spaceflight data ${ }^{4,5,36-38}$. A reduction of cardiac contractility is also in general accordance with cardiac atrophy observed during bed rest ${ }^{39-41}$. CO drop $(-7.58 \%)$ is less evident than $S V$, due to the $H R$ increase in the spaceflight configuration ( $+13 \%$, see the Methods section). The CO decrease is in qualitative agreement with the variations observed with respect to $1 \mathrm{G}$ supine condition ${ }^{4,5,36-38}$. As previously mentioned, $\mathrm{CO}$ (together with SV) is one of the cardiac parameters whose variation in $0 \mathrm{G}$ greatly depends on the chosen $1 \mathrm{G}$ baseline configuration. As observed by ref. ${ }^{12,42}$, in $\mathrm{OG}$ CO increased with respect to the sitting $1 \mathrm{G}$ position. Compared to the supine $1 \mathrm{G}$ position, $C O$ was usually observed to decrease $4,5,36-38$, although mild increases were also reported ${ }^{42}$. Mean central arterial pressure (MAP) was also tested for validation: MAP decreased by $9.92 \%$ in our model (as does brachial arterial pressure), falling within the range of measured data during long-term spaceflights ${ }^{4,6,43,44}$. Mild reduction of $M A P$ with respect to seated preflight values was also observed in long-term spaceflight using a countermeasure protocol $^{1,12}$. The green rows of Table 1 show that there is satisfactory agreement between currently available in vivo and our in silico outcomes. This confirms the correct $0 \mathrm{G}$ configuration setting and the model's accuracy. On the other hand, the scarcity of previously measured parameters and the good predictive level of the model justifies its use to obtain cardiac estimates not previously measured directly. It should be recalled that these cardiac variables were not used to tune the model parameters, but to a posteriori verify the modeling response once the parameter setting was completed. The comparison with the reported literature represents the only validation (with data actually measured and related to the same simulated configuration) currently available in $0 \mathrm{G}$ conditions.

Other cardiac parameters were also modeled. A substantial decrease of the work done by the heart $(-19.29 \%$ for SW/min) and a more limited reduction of oxygen consumption $(-1.91 \%$ for RPP, $-8.38 \%$ for $T \mathrm{TI} / \mathrm{min}$ ) were found. The slight decrease of myocardial oxygen consumption indexes represents an additional modeling validation, in agreement with the maximal oxygen uptake reduction observed in long-term spaceflight ${ }^{45}$. Central venous pressure (CVP), estimated as the mean value of the superior and inferior vena cava pressure signals averaged over the beat, decreased by $5.08 \%$. This result is in qualitative agreement with the CVP reduction observed in early spaceflights ${ }^{12,46,47}$ and parabolic flights ${ }^{48,49}$, though it should be kept in mind that the time scales and physical mechanisms inducing CVP variations found in literature are not fully comparable to the present study. Central aortic pulse pressure, $P P_{A A}$, decreased $(-22.30 \%)$ due to a higher reduction of central aortic systolic pressure $(-13.39 \%)$, with respect to the diastolic pressure decrement $(-6.90 \%)$. In agreement with the pulse pressure behavior, central aortic augmentation index, $A I_{A A}$, also declined (-41.18\%).

The observed variations of cardiac work, oxygen consumption, and contractility indexes, as well as central mean and pulse 
Table 1. Modeled cardiac parameters for the supine $1 \mathrm{G}$ configuration and during long-term $0 \mathrm{G}$ spaceflight (green color is adopted when spaceflight data are available, yellow otherwise).

\begin{tabular}{|c|c|c|c|c|}
\hline Variable & Supine 1G & Spaceflight 0G & \% Variation & Literature data \\
\hline$V_{\text {lves }}[\mathrm{ml}]$ & 48.43 & 50.73 & $+4.75 \%$ & - \\
\hline$V_{\text {lved }}[\mathrm{ml}]$ & 123.96 & 112.43 & $-9.30 \%$ & {$[-5 \%,-13 \%]^{4,5,36-38}$} \\
\hline$S V[\mathrm{ml}]$ & 75.54 & 61.70 & $-18.32 \%$ & {$[-14 \%,-23 \%]^{4,36-38}$} \\
\hline$E F[\%]$ & 60.93 & 54.88 & $-9.93 \%$ & {$[-5 \%,-11 \%]^{4,5,36-38}$} \\
\hline$C O[1 / \mathrm{min}]$ & 5.67 & 5.24 & $-7.58 \%$ & {$[-11 \%,-18 \%]^{4,36-38}$} \\
\hline$S W /$ min $[\mathrm{J} / \mathrm{min}]$ & 80.01 & 64.58 & $-19.29 \%$ & - \\
\hline$C V P[\mathrm{mmHg}]$ & 6.49 & 6.16 & $-5.08 \%$ & - \\
\hline$P_{A A, \text { syst }}[\mathrm{mmHg}]$ & 121.03 & 104.82 & $-13.39 \%$ & - \\
\hline$P_{A A, \text { dias }}[\mathrm{mmHg}]$ & 69.99 & 65.16 & $-6.90 \%$ & - \\
\hline$M A P[\mathrm{mmHg}]$ & 87.01 & 78.38 & $-9.92 \%$ & {$[-2 \%,-10 \%]^{4,6,43,44}$} \\
\hline$T T I /$ min $_{[\mathrm{mmHg} s / \mathrm{min}]}$ & 2590 & 2373 & $-8.38 \%$ & - \\
\hline$R P P[\mathrm{mmHg} / \mathrm{min}]$ & 9078 & 8905 & $-1.91 \%$ & - \\
\hline$A I_{A A}$ & 0.17 & 0.10 & $-41.18 \%$ & - \\
\hline$P P_{A A}[\mathrm{mmHg}]$ & 51.04 & 39.66 & $-22.30 \%$ & - \\
\hline
\end{tabular}

$V_{\text {lves }}$ end-systolic left ventricular volume, $V_{\text {lved }}$ end-diastolic left ventricular volume, SV stroke volume, EF ejection fraction, CO cardiac output, SW/min stroke work per minute, $C V P$ central venous pressure, $P_{A A, \text { syst }}$ and $P_{A A, \text { dias }}$ systolic and diastolic ascending aortic pressures, MAP mean arterial pressure, $T T / /$ min tension time index per minute, $R P P$ rate pressure product, $A l_{A A}$ and $P P_{A A}$ augmentation index and pulse pressure for the ascending aortic district.

pressures signal an overall scenario of cardiac deconditioning. The cardiovascular system reaches a condition commonly seen in a sedentary lifestyle. This is achieved initially by direct fluid dynamics changes and is then maintained through continued presence in the less demanding $0 \mathrm{G}$ environment.

\section{Pressures, flow rates and volumes}

Pressure, $P(t)$, flow rate, $Q(t)$, and volume, $V(t)$, time-series were analyzed together with the relative variations of the beataveraged values $(\bar{P}, \bar{Q}$, and $\bar{V})$ during spaceflight with respect to the supine $1 \mathrm{G}$ configuration on the Earth. In addition to mean variation, relative variation of pulse pressure, $P P$ (defined, in the general case, as the difference between maximum and minimum pressure values), and maximum pressure values, $P_{\max }$, were evaluated. In addition to being general indicators of health and being mechanical properties of the cardiovascular system, these latter quantities estimate the vertical stretching of the pressure signals. Figure 1 shows representative results for the four macroregions considered, while $P$ and $Q$ variations at other significant sites are listed in Tables 2 and 3.

Both pressure and flow rate mean levels decreased in the spaceflight configuration. Pressure variations were quite homogeneous throughout the different cardiovascular regions, ranging between -8 and $-12 \%$ (apart from the cardiac region and venous return, where variations are lower, between -2 and $-6 \%$ ). Flow rate changes were more heterogeneous. In particular, variations of the mean values decreased from the upper to the lower body, from -16 to $-18 \%$ in the cerebral region to -2 to $-6 \%$ in the lower limbs (apart from the anterior tibial artery, $-11.94 \%$ ). Pressure maximum variations are more heterogeneous (around -5 to $-15 \%$ ) than mean levels, and they are both smaller in the venous return than in the arterial circulation. $P P$ variations remarkably showed high variability (ranging from +4.82 to $-56.00 \%)$, which is not directly attributable to the location, type (venous/arterial), and size of the region. An example is represented by the superior and inferior venae cavae (see Table 3), both veins ending in the right atrium and similar in size, but showing completely different $P P$ variations ( +4.82 and $-32.97 \%)$.
Volume time-series, $V(t)$, and relative variations of the average values per beat $(\bar{V})$ during spaceflight with respect to the supine $1 \mathrm{G}$ condition are reported in Fig. 2 for the $\mathrm{OD}$ compartments. Mean pressure and flow rate values are strictly related to blood volume variations induced by the prolonged exposure to microgravity, since $V=V_{0}+C P$, where $C$ and $V_{0}$ are the compliance and unstressed volume of each district, respectively. In the lower limbs and abdominal region below VIP (VIP, see the Methods for more details), mean volume variations were widely negative. In the upper body, volume reduction was smaller, resulting in volume increase in the upper body and pulmonary regions. The different upper-to-lower body volume repartition primarily resulted from the blood shift, which in the upper body partially counterbalances the effects of volume reduction. The further cardiac volume and contractility reduction mitigated the fluid shift effects in the cardiac region. As a result, heart chamber volumes were reduced more than the thoracic surroundings.

The time-series of the hemodynamic variables shown in Figs. 1 and 2 demonstrate the whole signal differences between $1 G$ supine and $0 G$ conditions. Signals were not merely translated or scaled but, also due to the different $H R$, their waveforms changed. This aspect is discussed in more detail in the next section.

\section{Hemodynamic signals: waveform alteration}

We focused on the waveform alteration of pressure and flow rate signals in terms of the normalized signal difference, NSD, which is a dimensionless measure of signaling similarity (ranging in the interval [0.2]) between the $1 G$ supine and $0 G$ spaceflight configurations (see the Methods section for more details). Representative results of each cardiovascular region are shown in Fig. 3, while NSD values for both $P^{\prime}$ and $Q^{\prime}$ at different sites can be found in Table 4.

It is immediately apparent that the normalized signals $P^{\prime}$ e $Q^{\prime}$ in OG (red curves) in all cardiovascular sites were delayed with respect to the $1 \mathrm{G}$ supine condition (blue curves), as a result of the horizontal stretching due to the faster $R R$ beating rate in OG. However, the delay was not merely due to $H R$ variations, as it was not uniformly found in all the regions, but varied without showing 

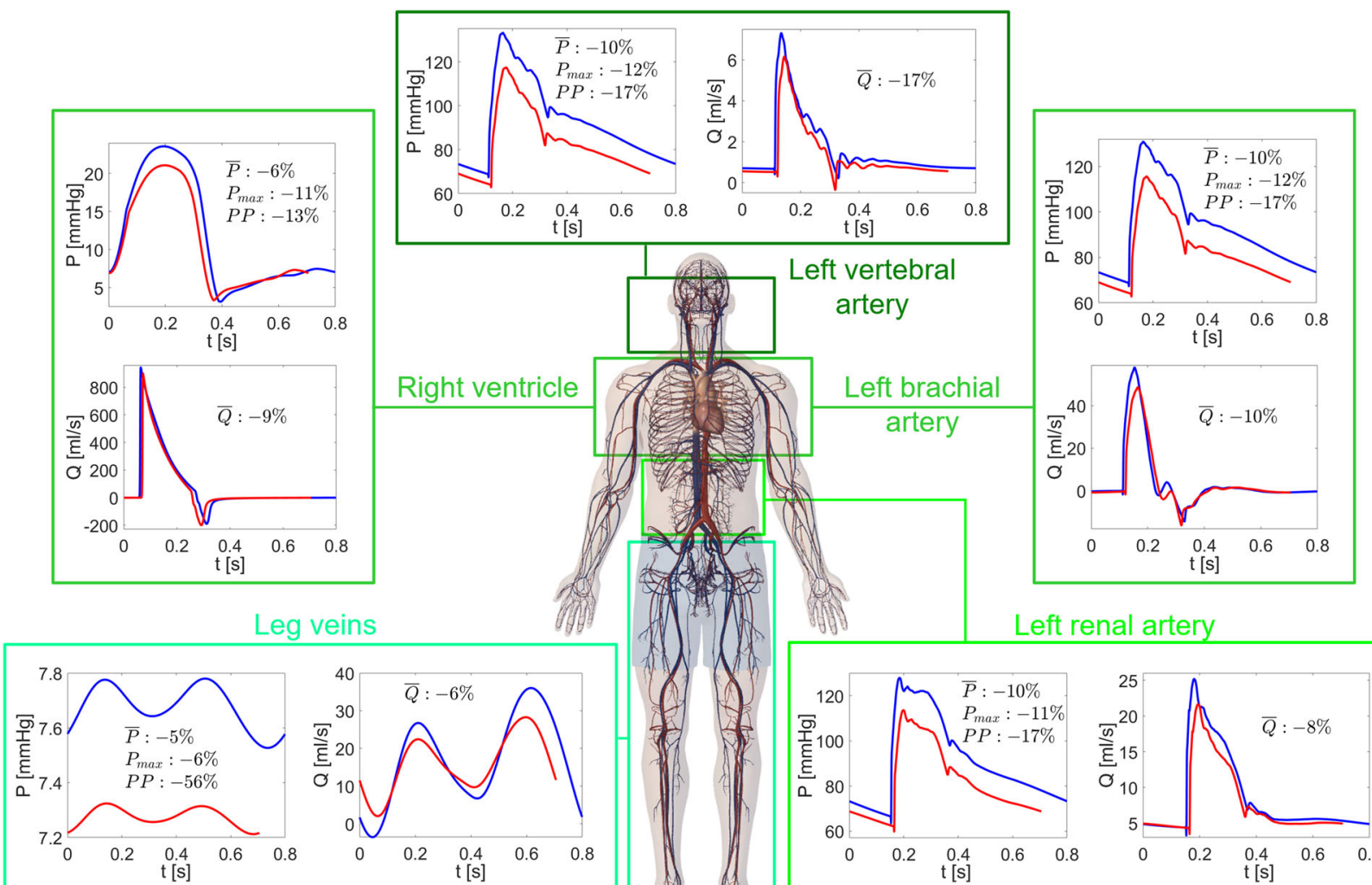

g veins
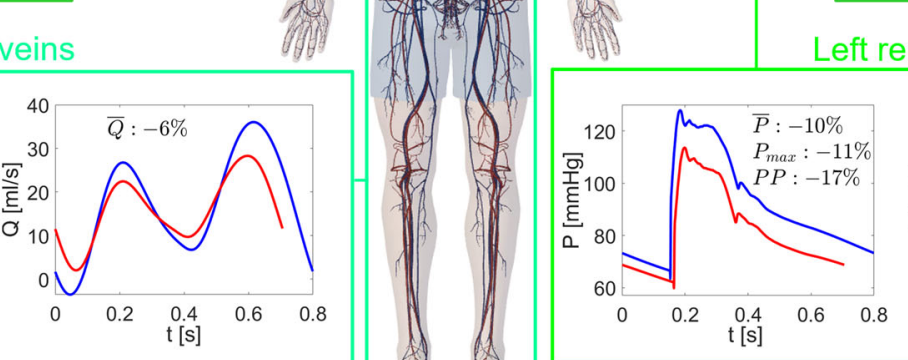

eft renal artery

Fig. 1 Pressures and flow rates throughout the body in $1 \mathbf{G}$ supine and $\mathbf{0 G}$ conditions. Time-series of the pressure, $P(t)$, and flow rate, $Q(t)$, at different sites: left vertebral artery (cerebral), right ventricle and left brachial artery (cardio-thoracic region), left renal artery (abdominal region), leg veins (lower limbs). Blue: supine 1G configuration on the Earth; red: 0G spaceflight configuration. Mean pressure $(\bar{P})$, mean flow rate $(\bar{Q})$, together with maximum pressure $\left(P_{\max }\right)$, and pulse pressure $(P P)$ relative variations between $1 \mathrm{G}$ and $0 \mathrm{G}$ configurations are reported in each panel.

a significant trend from upper to lower regions. This means that waveform alteration acts differently throughout the body and as well as at the same site. For instance, by comparing $0 \mathrm{G}$ and $1 \mathrm{G}$, note the different shape and phase of the $Q^{\prime}$ signals in the inferior vena cava, and the different oscillation amplitude for the carotid $P^{\prime}$ and $Q^{\prime}$ signals. NSD, which was introduced to quantify signal stretching besides pure horizontal translation, was generally not negligible and often beyond 0.5 . Flow rates were more affected by shape variations than pressures, and proximal regions were less prone to waveform variations than distal regions (see Table 4).

Arterial proximal-to-distal tree

Special attention was given to the arterial proximal-to-distal tree, focusing on the arterial 1D system from the ascending aorta to the anterior tibial artery. The scenario emerging from Fig. 4 (and detailed in Tables 2 to 4 ) is multi-faceted and not always ascribable to the macro-regions crossed by the pathway. Mean flow rate variations were close to $-9 \%$ in the ascending aorta, increasing to approximately $-6 \%$ in the last part of the abdominal aorta, and then decreasing again towards the anterior tibial artery to around $-12 \%$. Mean pressure variations were instead quite constant, in the range of $-10 \%$, along the pathway, similarly to what happens for the $P_{\max }(-11$ to $-13 \%)$. The overall proximal-to-distal trend of $P P$ variations was a slight decrease, while NSD variations increased remarkably. In particular, NSD at least doubled for $P^{\prime}$ and $Q^{\prime}$, and $Q^{\prime}$ variations were also larger than for $P^{\prime}$. What was previously reported for the $P^{\prime}, Q^{\prime}$, and NSD behavior from proximal-to distal areas is also true here, where only arterial sites were considered.

\section{DISCUSSION}

These results depict a complex picture where changes imposed by the $O G$ configuration created remarkably heterogenous stresses on the hemodynamic variables of the cardiovascular system. Volume reduction and baroreflex responses acted on global levels of pressure, volume, and flow rate, while blood shift, resistance and compliance variations altered the local mean levels of the hemodynamic variables throughout the body. The most important alteration in volume was the combination of blood shift and reduction: in the upper body the incoming blood volume partially compensated for the volume lost, resulting in either small reductions or positive variations (in particular, at the pulmonary and cerebral levels). In the lower body two contributions added up, leading to a more evident volume decrease. However, the upper body blood volume overload did not translate into pressure and flow rate increases due to the complex interplay between resistance and compliance variations within each region. Moreover, it should be kept in mind that (negative) variations were referred to the $1 \mathrm{G}$ supine condition, while in the cerebral circulation positive variations are expected if referred to the $1 \mathrm{G}$ standing position, as suggested by ref. ${ }^{50}$. The increased venous compliance observed in the legs confined lower body mean pressure variations to a range which is similar to that of the upper body, that is -8 to $-12 \%$ (see Fig. 1 and Table 2). The smallest variations $(-5 \%)$ occurred in the capillary-venous districts of the legs. Cardiac compliance increased accounting for the reduced contractility, and this in turn limited pressure variations at the central level ( -1 to $-8 \%)$. If arterial resistance had not changed, following homogeneous pressure variations we would have expected homogeneous flow 


\begin{tabular}{|c|c|c|c|c|c|c|}
\hline Cardiovascular region & \multicolumn{3}{|c|}{ Pressure $\bar{P}[\mathrm{mmHg}]$} & \multicolumn{3}{|c|}{ Flow rate $\bar{Q}[\mathrm{ml} / \mathrm{s}]$} \\
\hline \multicolumn{7}{|l|}{ Arterial tree pathway (1D) } \\
\hline Ascending aorta & 92.61 & 83.19 & $-10.17 \%$ & 87.85 & 80.01 & $-8.92 \%$ \\
\hline Abdominal aorta $\mathrm{E}$ & 90.76 & 81.45 & $-10.26 \%$ & 17.01 & 16.02 & $-5.82 \%$ \\
\hline External iliac & 90.48 & 80.93 & $-10.55 \%$ & 6.31 & 5.86 & $-7.13 \%$ \\
\hline Femoral & 89.47 & 80.20 & $-10.36 \%$ & 3.24 & 2.94 & $-9.26 \%$ \\
\hline \multicolumn{7}{|l|}{ Cerebral region (OD-1D) } \\
\hline HA veins & 6.94 & 6.54 & $-5.76 \%$ & 20.82 & 17.86 & $-14.22 \%$ \\
\hline Left internal carotid arterioles & 80.40 & 73.32 & $-8.81 \%$ & 2.18 & 1.78 & $-18.35 \%$ \\
\hline Left external carotid & 92.28 & 83.05 & $-10.00 \%$ & 2.34 & 1.90 & $-18.80 \%$ \\
\hline Left external carotid arterioles & 80.37 & 73.32 & $-8.77 \%$ & 2.35 & 1.93 & $-17.87 \%$ \\
\hline \multicolumn{7}{|l|}{ Cardio-thoracic region (OD-1D) } \\
\hline LA and MV & 7.51 & 7.39 & $-1.60 \%$ & 87.91 & 80.11 & $-8.87 \%$ \\
\hline LV and AV & 43.20 & 39.59 & $-8.36 \%$ & 87.85 & 80.01 & $-8.92 \%$ \\
\hline RA and TV & 6.47 & 6.14 & $-5.10 \%$ & 87.90 & 80.15 & $-8.82 \%$ \\
\hline RV and PV & 11.64 & 10.95 & $-5.93 \%$ & 87.84 & 80.04 & $-8.88 \%$ \\
\hline Pulmonary arteries & 15.42 & 14.59 & $-5.38 \%$ & 87.83 & 80.02 & $-8.89 \%$ \\
\hline Pulmonary veins & 8.39 & 8.19 & $-2.38 \%$ & 87.86 & 80.05 & $-8.89 \%$ \\
\hline SVC & 6.48 & 6.15 & $-5.09 \%$ & 20.80 & 17.85 & $-14.18 \%$ \\
\hline Gastric arterioles & 48.88 & 44.05 & $-9.88 \%$ & 4.92 & 4.42 & $-10.16 \%$ \\
\hline Celiac A & 91.57 & 82.26 & $-10.17 \%$ & 10.17 & 9.19 & $-9.63 \%$ \\
\hline Left renal & 90.43 & 81.23 & $-10.17 \%$ & 8.50 & 7.81 & $-8.12 \%$ \\
\hline $\mathrm{U}$ left renal arterioles & 66.18 & 58.90 & $-11.00 \%$ & 4.19 & 3.68 & $-12.17 \%$ \\
\hline $\mathrm{L}$ left renal arterioles & 66.18 & 58.90 & $-11.00 \%$ & 4.30 & 4.13 & $-3.95 \%$ \\
\hline$U \mathrm{ABD}$ venules & 12.94 & 11.90 & $-8.04 \%$ & 26.35 & 23.44 & $-11.04 \%$ \\
\hline$L A B D$ venules & 12.98 & 12.38 & $-4.62 \%$ & 23.64 & 22.68 & $-4.06 \%$ \\
\hline \multicolumn{7}{|l|}{ Lower limbs region (0D-1D) } \\
\hline Inner iliac & 90.47 & 81.17 & $-10.28 \%$ & 2.20 & 2.15 & $-2.27 \%$ \\
\hline Inner iliac arterioles & 78.65 & 69.59 & $-11.52 \%$ & 2.20 & 2.15 & $-2.27 \%$ \\
\hline Deep femoral & 89.44 & 80.17 & $-10.36 \%$ & 3.08 & 2.93 & $-4.87 \%$ \\
\hline Deep femoral arterioles & 68.12 & 59.74 & $-12.30 \%$ & 3.07 & 2.93 & $-4.56 \%$ \\
\hline Anterior tibial & 84.44 & 75.61 & $-10.46 \%$ & 2.01 & 1.77 & $-11.94 \%$ \\
\hline Anterior tibial arterioles & 38.20 & 34.16 & $-10.58 \%$ & 2.01 & 1.78 & $-11.44 \%$ \\
\hline Posterior tibial & 84.90 & 76.00 & $-10.48 \%$ & 1.22 & 1.15 & $-5.74 \%$ \\
\hline Posterior tibial arterioles & 64.36 & 56.33 & $-12.48 \%$ & 1.23 & 1.16 & $-5.69 \%$ \\
\hline Legs capillaries & 27.13 & 25.63 & $-5.53 \%$ & 17.02 & 16.04 & $-5.76 \%$ \\
\hline Legs veins & 7.68 & 7.28 & $-5.21 \%$ & 17.03 & 16.04 & $-5.81 \%$ \\
\hline
\end{tabular}


Table 3. Maximum pressure, $P_{\max }$, and pulse pressure, $P P$, values $[\mathrm{mmHg}]$ at different significant sites in supine $1 \mathrm{G}$ and $0 \mathrm{G}$ conditions, with relative variations [\%].

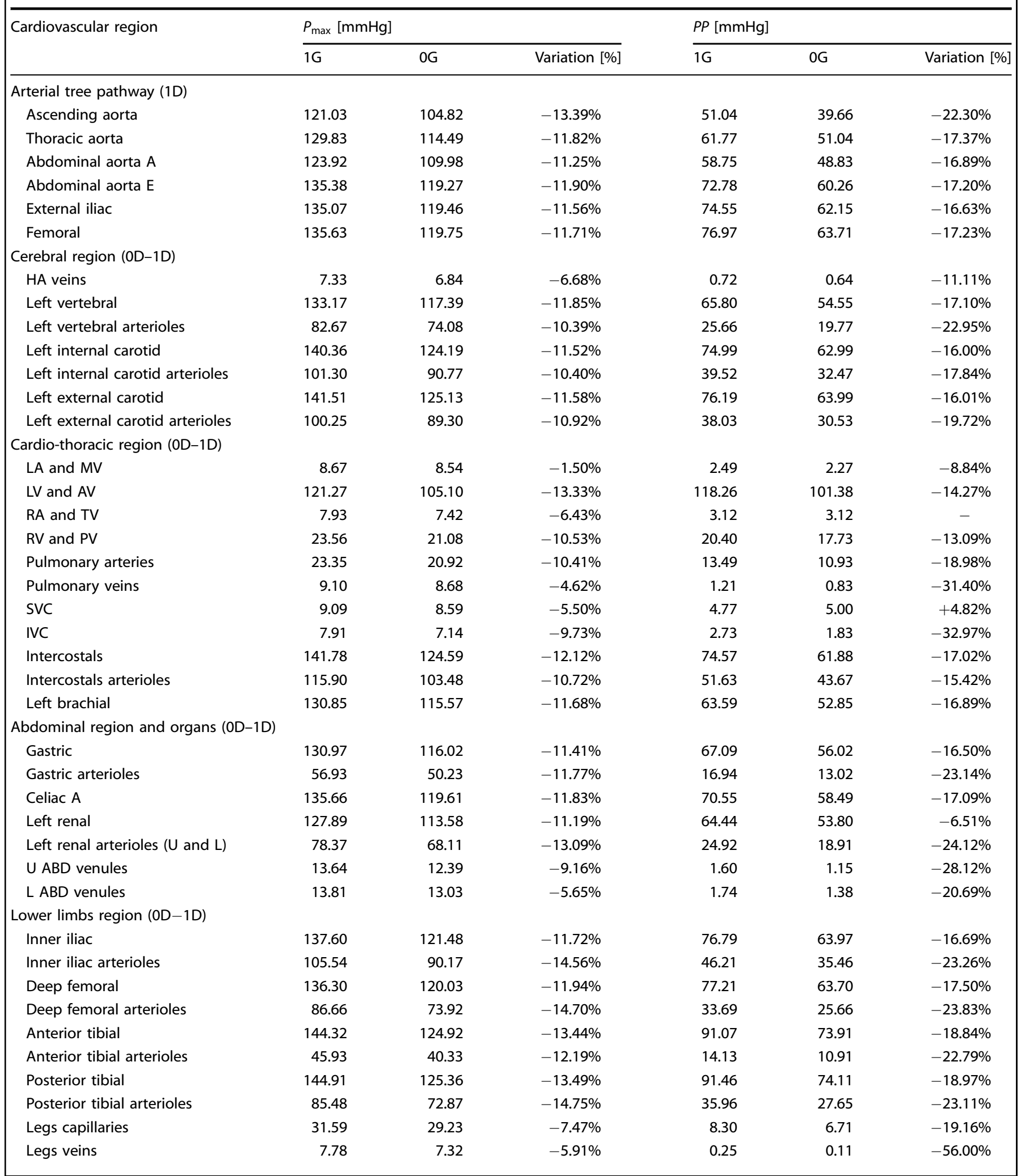

rate variations throughout the body, around -8 to $-12 \%$. Instead, all lower body resistances reduced, while carotid/ vertebral resistances increased. Therefore, flow rates towards upper body and cerebral areas were reduced $(-17$ to $-19 \%)$, while lower body flow rate was on average boosted ( -2 to $-5 \%$, with the anterior tibial artery as only exception). The cardiothoracic region experienced flow rate reductions in between lower and upper body values, namely -8 to $-10 \%$. 


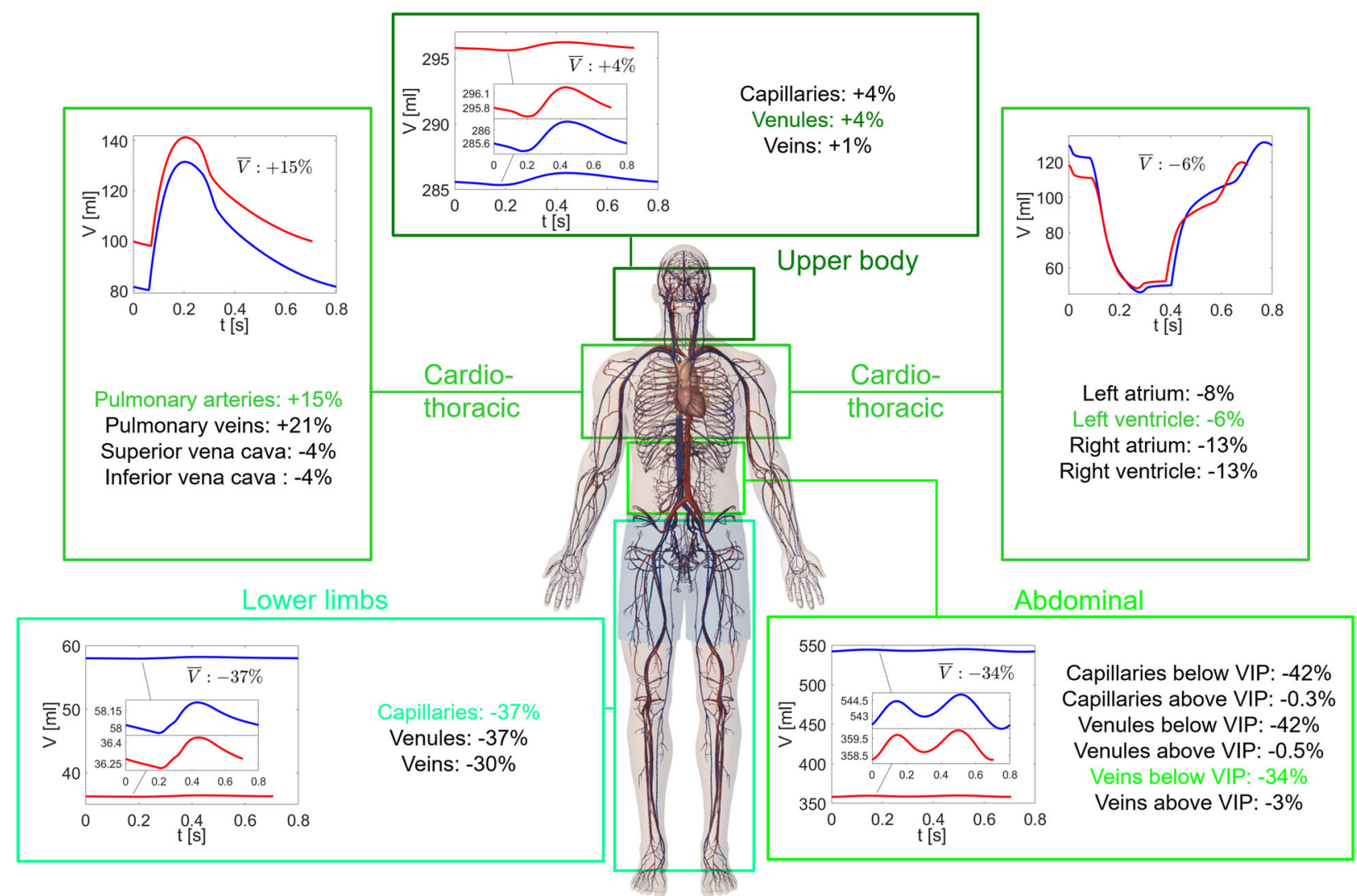

Fig. 2 Volumes throughout the body in 1 G supine and 0 G conditions. Time-series of the volume, $V(t)$, at different $0 D$ sites: venules (upper body), left ventricle and pulmonary artery (cardio-thoracic region), veins below VIP (abdominal region), capillaries (lower limbs). Blue: supine $1 \mathrm{G}$ configuration on the Earth; red: $0 \mathrm{G}$ spaceflight configuration. Mean volume $(\bar{V})$ relative variations between $1 \mathrm{G}$ and $0 \mathrm{G}$ configurations are reported for each region (colored values refer to the corresponding time-series shown).

Mean flow rate variations decreased from ascending aorta to the inferior abdominal aorta, consistent with what happened from upper towards lower body, i.e., due to resistance changes flow rate was more hampered in the upper thoracic region with respect to the lower abdomen. Note also that ascending aorta variations were damped by the cardiac volume and contractility reduction. Mean flow rate variations then increased again towards the inner iliac-femoral-posterior tibial path, for two possible reasons: (i) conduction 1D arteries (e.g., femoral and external iliac arteries) are less influenced by resistance variations than their bifurcating branches (e.g., deep femoral and inner iliac arteries); (ii) on the Earth, resistance of the posterior tibial artery is higher than the anterior tibial. Equal relative reduction of resistance $(-10 \%)$ for both arteries led to an absolute resistance reduction which is more important for the posterior tibial artery. In the latter, flow rate was thus more enhanced (i.e., lower mean $Q$ variations) than in the anterior tibial artery (higher mean $Q$ variations).

The hemodynamic and cardiac parameter variability reveals a pattern which, overall, resembles the spaceflight configuration of a relaxed, sedentary lifestyle. This is directly due to both fluid dynamics effects as well as reduced physical activity and lower cardiac work to counter the force of gravity ${ }^{3}$. Cardiac work, oxygen consumption, and contractility indexes were reduced, as were central mean and pulse pressures. Almost all the hemodynamic and cardiac parameters resulted from variables pertaining to the thoracic-cardiac region, for which the above considerations about $P$ and $Q$ mean values still hold. The hemodynamic mechanisms mainly responsible for the observed cardiac deconditioning are the global volume reduction together with the decrease of cardiac volume and contractility. The slight increase of $V_{\text {lves }}$ can be interpreted as a possible sign of a reduced ability of the ventricle to empty and ventricular overload due to fluid shift (pulmonary artery and vein volumes increased on average by $14.61 \%$ and $21.39 \%$, respectively).

Maximum and mean pressure value variations were smaller in the venous than in the arterial compartments (see Tables 2 and 3). This behavior is related to the higher compliance of the venous regions, which was further increased in the lower limbs during spaceflight. Venous vessels were thus more able to absorb and reduce pressure changes than their arterial counterparts. In general, PP variations were higher than mean pressure and $P_{\max }$ changes: apart from one exception $(+4.82 \%)$, pressure signals tended to reduce their ranges to a greater extent than their means. In general signals were less pulsatile (between -10 and $-30 \%$ from $1 \mathrm{G}$ supine to $0 \mathrm{G}$ ) and, in each region, the greatest $P P$ variations (e.g., $>20 \%$ ) usually occurred in the distal areas, where $P P$ on the Earth is already limited and among the lowest in that region: arterioles, venules and veins were thus the most affected areas. However, the contrary was not in general true: the smallest $P P$ values on the Earth (e.g., right atrium and superior vena cava) did not necessarily imply the largest $P P$ variations during spaceflight.

$P P$ and NSD variations showed great variability throughout the body, meaning that signals were stretched differently in vertical and horizontal directions depending on the site. PP and NSD alterations were inverted along the 1D arterial tree (see Fig. 4 and Tables 3, 4). At one extreme (ascending aorta) PP variation was high and signal was more squeezed than in distal regions, but NSD variations were limited. At the other extreme (anterior tibial artery), pressure signal was stretched less but experienced a remarkable waveform change in terms of NSD. 


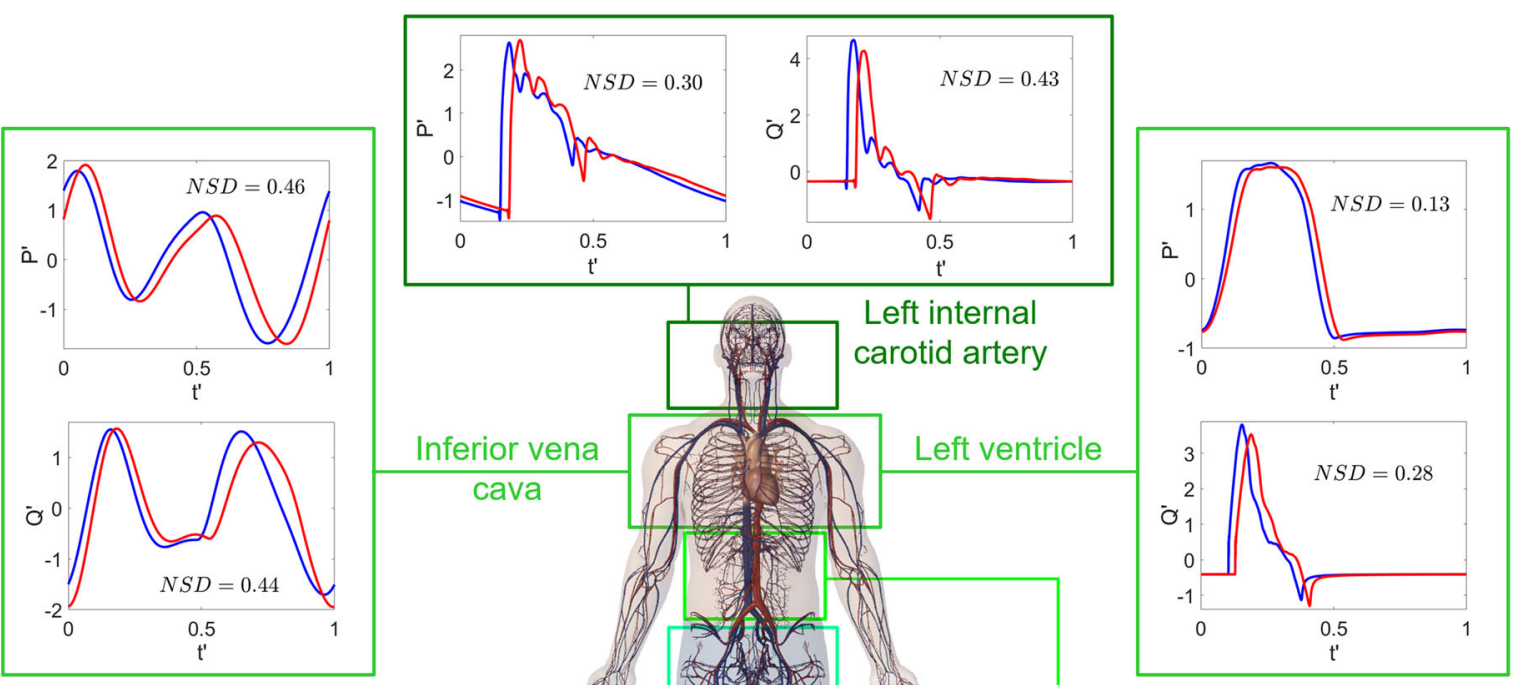

Posterior tibial artery
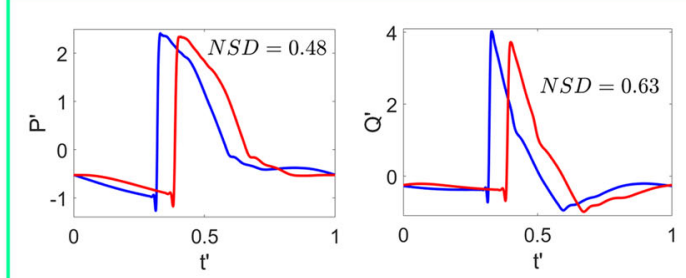

Q60.

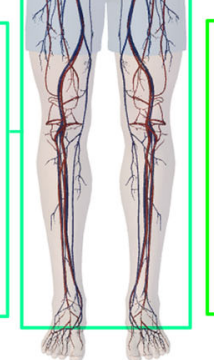

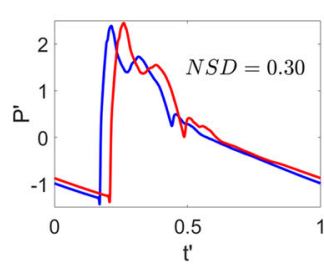

Celiac artery

Fig. 3 Pressure and flow rate waveform alterations. Normalized time-series $P^{\prime}\left(t^{\prime}\right)$ and $Q^{\prime}\left(t^{\prime}\right)$ at different sites: left internal carotid artery (cerebral), left ventricle and inferior vena cava (cardio-thoracic region), celiac artery (abdominal region), posterior tibial artery (lower limbs). Blue: supine 1G configuration on the Earth; red: 0G spaceflight configuration. NSD (normalized signal difference) values for $Q^{\prime}$ and $P^{\prime}$ are reported in each panel.

A possible interpretation for the NSD increase towards the distal regions is that $0 \mathrm{G}$-induced waveform alterations mainly happen peripherally, where the traveling wave is already modified in $1 \mathrm{G}$ by vessel tapering and non-linearities ${ }^{51}$, and the great majority of reflections occur ${ }^{52}$. In addition, since distal regions are most closely influenced by arterial resistance and venous compliance variations, more pronounced alterations can be expected there. Thus, in the proximal regions the interaction of many backward waves arriving at different times makes them dampen and partially elide each other. As a result, the total wave was shaped mainly by the forward contribution, and waveform variation induced by the $0 \mathrm{G}$ configuration was less evident $(N S D<0.2)$. When instead, in the peripheral zones, forward and backward waves are comparable due to the proximity to reflection sources (such as bifurcations, discontinuities, or arteriolar interface), NSD was up to or beyond 0.5 . The backward wave being most influenced during the $0 G$ configuration explains the greater NSD variations for $Q$ than $P$. In fact, the flow rate backward wave is upside down with respect to the corresponding forward wave, while the pressure backward wave is reflected upright. Thus, the same backward wave variation (in absolute amplitude) has a stronger impact on the total flow rate wave rather than on pressure.

The NSD increase observed in the peripheral regions is likely due to reflection mechanisms within the traveling wave combined with the $H R$ increase which, during spaceflight, exacerbated the waveform alteration. Moreover, due to the $H R$ increase in $0 G$ the systolic interval was longer (with respect to the $R R$ period) than in $1 G$. Even if the imposed HR increase was not worrisome $(+13 \%)$, diastole was shortened and diastolic function (e.g., ventricular filling) could be partly affected. As previously noted, waveform variations induced by reflection mechanisms were not absorbed downstream in the arterial tree, leading to evident signal variations (e.g., NSD values around $0.4-0.5$ ) in arteriolar-capillary districts, leg veins, and venae cavae. It is reasonable to expect that these regions downstream of the arterial system do nothing but undergo and maintain the changes introduced upstream. The normal wave pattern was therefore modified and all the physiological phenomena at the capillary-venous level-such as regular perfusion, mean pressure per beat, and average nutrient supply at the cellular level-can experience important alterations.

Despite the fact that hemodynamic changes are important and diversified the observed variations in the steady-state $0 \mathrm{G}$ spaceflight configuration are not dramatic per se. For instance, although $E F$ reduction was not negligible $(-10 \%)$, myocardial contractility reduction is considered clinically relevant when $E F$ drops $20 \%$ or more, which has not been observed to date during any spaceflight $^{53}$. After long-term spaceflight a OG adaptation point imposed by a less demanding environment is indeed reached by the cardiovascular system. The scenario becomes potentially hazardous at the time of reentry on the Earth or partial gravity restoration (e.g., Moon/Mars landing). Leaving the OG environment imparts a backward blood shift from upper-to-lower body. Recalling that mean pressure at central, cerebral, and organ levels was decreased by $10 \%$, backward blood shift combined with an overall hypovolemic condition $(-11.5 \%$ for the total blood volume) can impair normal perfusion above VIP. Moreover, compliance increase and resistance decrease in the lower limbs amplify the venous pooling already present during reentry and landing. All these mechanisms converge to produce the wellknown condition of orthostatic intolerance, which is further aggravated by the reduced and slowed baroreceptor response. In fact, as baroreceptors are not able to promptly control the 
Table 4. NSD values at different significant sites for pressure and flow rate signals.

\begin{tabular}{|c|c|c|}
\hline Cardiovascular region & NSD pressure & NSD flow rate \\
\hline \multicolumn{3}{|l|}{ Arterial tree pathway (1D) } \\
\hline Ascending aorta & 0.19 & 0.28 \\
\hline Thoracic aorta & 0.24 & 0.37 \\
\hline Abdominal aorta $\mathrm{A}$ & 0.31 & 0.37 \\
\hline Abdominal aorta $\mathrm{E}$ & 0.33 & 0.47 \\
\hline External iliac & 0.34 & 0.49 \\
\hline Femoral & 0.37 & 0.56 \\
\hline \multicolumn{3}{|l|}{ Cerebral region (0D-1D) } \\
\hline HA veins & 0.56 & 0.56 \\
\hline Left vertebral & 0.25 & 0.32 \\
\hline Left vertebral arterioles & 0.25 & 0.23 \\
\hline Left internal carotid & 0.30 & 0.43 \\
\hline Left internal carotid arterioles & 0.26 & 0.27 \\
\hline Left external carotid & 0.30 & 0.41 \\
\hline Left external carotid arterioles & 0.23 & 0.25 \\
\hline \multicolumn{3}{|l|}{ Cardio-thoracic region (OD-1D) } \\
\hline LA and MV & 0.26 & 0.34 \\
\hline LV and AV & 0.13 & 0.28 \\
\hline RA and TV & 0.41 & 0.44 \\
\hline RV and PV & 0.15 & 0.23 \\
\hline Pulmonary arteries & 0.16 & 0.17 \\
\hline Pulmonary veins & 0.31 & 0.23 \\
\hline SVC & 0.51 & 0.49 \\
\hline IVC & 0.46 & 0.44 \\
\hline Intercostals & 0.29 & 0.49 \\
\hline Intercostals arterioles & 0.26 & 0.28 \\
\hline Left brachial & 0.25 & 0.42 \\
\hline \multicolumn{3}{|c|}{ Abdominal region and organs (OD-1D) } \\
\hline Gastric & 0.31 & 0.32 \\
\hline Gastric arterioles & 0.28 & 0.31 \\
\hline Celiac A & 0.30 & 0.39 \\
\hline Left renal & 0.27 & 0.32 \\
\hline $\mathrm{U}$ left renal arterioles & 0.30 & 0.33 \\
\hline$L$ left renal arterioles & 0.30 & 0.30 \\
\hline$U A B D$ venules & 0.30 & 0.30 \\
\hline$L A B D$ venules & 0.33 & 0.33 \\
\hline \multicolumn{3}{|l|}{ Lower limbs region (0D-1D) } \\
\hline Inner iliac & 0.34 & 0.48 \\
\hline Inner iliac arterioles & 0.34 & 0.35 \\
\hline Deep femoral & 0.37 & 0.48 \\
\hline Deep femoral arterioles & 0.38 & 0.37 \\
\hline Anterior tibial & 0.48 & 0.54 \\
\hline Anterior tibial arterioles & 0.45 & 0.60 \\
\hline Posterior tibial & 0.48 & 0.63 \\
\hline Posterior tibial arterioles & 0.48 & 0.52 \\
\hline Legs capillaries & 0.40 & 0.40 \\
\hline Legs veins & 0.50 & 0.50 \\
\hline
\end{tabular}

vasculature and regulate chronotropic and inotropic effects, a normotensive state is hardly guaranteed ${ }^{3}$.

Exercise capability was also markedly reduced. A hypothetical Moon or Mars landing (with partial gravity restored) requires a high work capacity and exercise tolerance, which were instead damped with respect to preflight conditions. The heart got used to a reduced pumping requirement and reduced work, with subsequent need for less oxygen. The less demanding spaceflight environment caused a central $P P$ reduction of $22.30 \%$. In $1 G$ conditions $P P$ is lower for sedentary than resistance-trained people ${ }^{54-57}$, thus $P P$ can be taken as a marker of training level and physical activity. Gravity is itself a natural source of loading for the human body: the lower spine, lower extremities, and postural muscles are loaded up to 4-5 times the body weight simply walking on the Earth ${ }^{2}$. Therefore, in 1G, strength training is naturally provided by the gravity vector on the body. During spaceflight this natural load is absent and, without any particular countermeasure program, the exercise tolerance of a spaceflight traveler becomes comparable to that of an untrained person with a sedentary lifestyle.

All these aspects, along with other spaceflight-induced effects such as muscular atrophy and bone demineralization, need to be properly addressed especially in long-term human space missions where prompt physical capability after partial or complete gravity restoration is required. The outcomes of the present study highlighted a wealth of cardiovascular information otherwise not yet available in vivo, showing how computational hemodynamics is particularly valuable in environmental conditions such as microgravity and spaceflight where even basic clinical measures are rarely available. It should be also noted that given identification (sex and age) and anthropometric parameters (such as weight and height), together with basic cardiovascular information (such as brachial cuff pressure), the model can be made patient-specific. That would provide good estimates of cardiovascular fitness related to the heterogeneity of the crew, similar to what was already done in $1 \mathrm{G}$ physiological and pathological conditions ${ }^{32,34,58}$. Cardiovascular deconditioning without ad hoc countermeasures, as modeled here, is of fundamental importance to have a clear and valid baseline for testing against the cardiovascular response with specific countermeasures. By comparing and contrasting the two conditions the efficacy of a single or a combination of countermeasures can be extensively evaluated. A future step might include the effect of one or more countermeasures in the model to observe the response to and help design, implement, and improve such countermeasures. Future modeling developments can also estimate the grade of orthostatic intolerance and exercise tolerance by simulating the return to a gravitational environment, starting from the cardiovascular deconditioned configuration here described. Eventually, understanding the mechanisms of cardiovascular deconditioning during spaceflight will have a significant impact on the knowledge of aging physiology on the Earth ${ }^{3}$. Since deconditioning in spaceflight by gravity deprivation is strongly analogous to deconditioning on the Earth by gravity withdrawal, as in sedentary aging, long-term spaceflight physiology can offer precious hints for delaying or preventing modern lifestyle medical disorders related to increased longevity.

\section{METHODS}

\section{Mathematical model}

The present multiscale modeling of the cardiovascular system combines a 1D description of the arterial tree together with a lumped parameterization of the remaining regions, that is, venous return, heart chambers, pulmonary circulation and baroreceptor regulation (see Fig. 5). The model has been tested using heart pacing and open-loop response, resulting in good agreement with measured data ${ }^{31}$. The hemodynamics of large-tomedium sized arteries relies on the model first proposed and validated by Guala et al. $^{32}$, which has been employed to study aging ${ }^{24,25}$ and atrial fibrillation effects ${ }^{28,29}$.

Mass and momentum balance equations were expressed in onedimensional form, considering dissipation and inertial terms, and were combined with a constitutive equation accounting for the nonlinear 


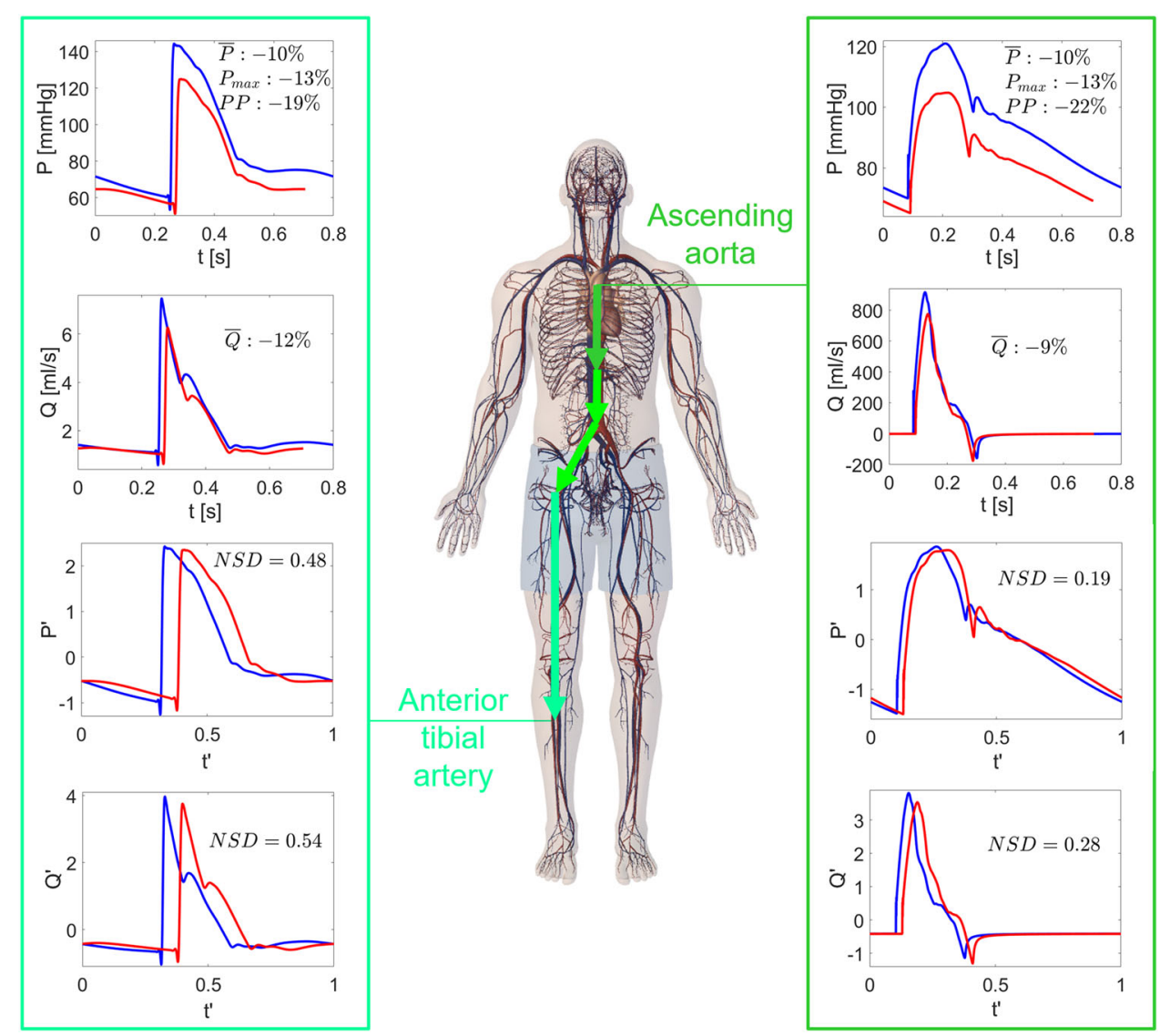

Fig. 4 Proximal-to-distal arterial tree: pressures and flow rates in $\mathbf{1 G}$ supine and $\mathbf{0 G}$ conditions. Time-series $P(t), Q(t), P^{\prime}\left(t^{\prime}\right)$, and $Q^{\prime}\left(t^{\prime}\right)$ at the extremes of the 1D arterial proximal-to-distal tree: ascending aorta and anterior tibial artery. Blue: supine $1 \mathrm{G}$ configuration on the Earth; red: OG spaceflight configuration. NSD values for $Q^{\prime}$ and $P^{\prime}$, together with mean pressure $(\bar{P})$, mean flow rate $(\bar{Q})$, max pressure $\left(P_{\max }\right)$, and pulse pressure $(P P)$ relative variations between $1 \mathrm{G}$ and $0 \mathrm{G}$ configurations are reported in the panels.

viscoelastic behavior of the tapered arterial walls. Arterioles, capillaries, venous return (venules, veins, and venae cavae), cardiac, and pulmonary (arterial and venous) circulations were modeled through RLC OD compartments, each characterizing local hemodynamics. The $R L C$ network accounts for viscous (resistance, $R$ ), inertial (inductance, $L$ ), and distensibility/contractility (compliance, $C$, or elastance, $E$ ) effects. All four cardiac chambers were contractile, as modeled through time-varying elastance functions, including the atrial kick feature. Valvular dynamics were well captured, dominated by the main forces acting on the valves, such as pressure difference across the valve, frictional effects from neighboring tissue resistance, the dynamic motion effect of the blood acting on the valve leaflet and the action of the vortex downstream of the valve. The 1D $\rightarrow O \mathrm{D}$ interface was at the arteriolar level, with a characteristic impedance per artery put before the corresponding arteriole. The $0 D \rightarrow 1 D$ interface was at the aortic valve level. The position of the volume indifference point (VIP, see the Supplementary Information for more details) split the model into upper and lower body regions (see Fig. 5). The short-term baroregulation mechanisms were also included in the model. The chronotropic and inotropic effects of both ventricles, as well as the control of the systemic vasculature (peripheral arterial resistances, unstressed volumes of the venous system, and venous compliances), were taken into account.

Simulations were run until the steady-state solution was achieved: for both $1 G$ supine and $O G$ conditions the transient dynamics were completely extinguished after 100 cardiac periods. Thus, results are referred to the generic steady-state $R R$ beat. Details of the governing equations and model parameters are offered in the Supplementary Information.

Definition and nomenclature for the common cardiac parameters are provided in the Supplementary Information. We defined a new metric in the present study, NSD. For the waveform analysis of signals, we exploited the dimensionless time $t^{\prime}=t / R R\left(t^{\prime} \in[0,1]\right)$, where $R R[\mathrm{~s}]$ is the cardiac beating period. We normalized the time-series as $y^{\prime}\left(t^{\prime}\right)=\left(y\left(t^{\prime}\right)-\mu_{y}\right) / \sigma_{y}$, where $\mu$ and $\sigma$ are the mean and standard deviation values of $y(t)$ over the cardiac beat, respectively $(y(t)=P(t)$ or $Q(t))$. We then defined the NSD averaged per heartbeat as

$$
\mathrm{NSD}=\int_{0}^{1}\left|y^{\prime}\left(t^{\prime}\right)_{1 \mathrm{G}}-y^{\prime}\left(t^{\prime}\right)_{0 \mathrm{G}}\right| d t^{\prime}, \quad \mathrm{NSD} \in[0,2],
$$

where $y^{\prime}\left(t^{\prime}\right)$ can be either $Q^{\prime}\left(t^{\prime}\right)$ or $P^{\prime}\left(t^{\prime}\right)$ (having zero mean and unitary standard deviation values), and the subscripts $1 G$ and $O G$ refer to the supine $1 \mathrm{G}$ and $O \mathrm{G}$ spaceflight configurations, respectively. As hemodynamic time-series $P^{\prime}\left(t^{\prime}\right)$ and $Q^{\prime}\left(t^{\prime}\right)$ were normalized in vertical (amplitude) and horizontal (time) directions, signals in $1 \mathrm{G}$ and $\mathrm{OG}$ can be fully compared: NSD quantitatively accounts for the shape and net waveform variation between them. The lower bound value $(N S D=0)$ is reached when the two signals overlap. Thus, if NSD is close to 0 , no appreciable waveform variation is detected. On the contrary, the upper bound value $(N S D=2)$ can be reached only if the two signals are step functions (centered in $t^{\prime}=$ 0.5 and $y^{\prime}=0$ and with half-width equal to 1 ), one complementary to the other. The closer NSD is to 2, the more significant is the alteration of the waveform.

Spaceflight setting: criteria, cardiovascular mechanisms, and adopted changes

The setting reproducing simulated long-term (at least 5 months) spaceflight conditions without ad hoc countermeasures relies on an extensive bibliographic investigation of more than 50 studies (listed in the Supplementary Information) of human cardiovascular changes induced by microgravity. A general overview of the adopted hemodynamic OG setting is sketched, while a detailed description is offered in the Supplementary Information. Among hemodynamic data available in literature we focused on those measured during spaceflights rather than ground-based experiments. When no useful information during actual spaceflight was available-such as for blood shift-we considered 


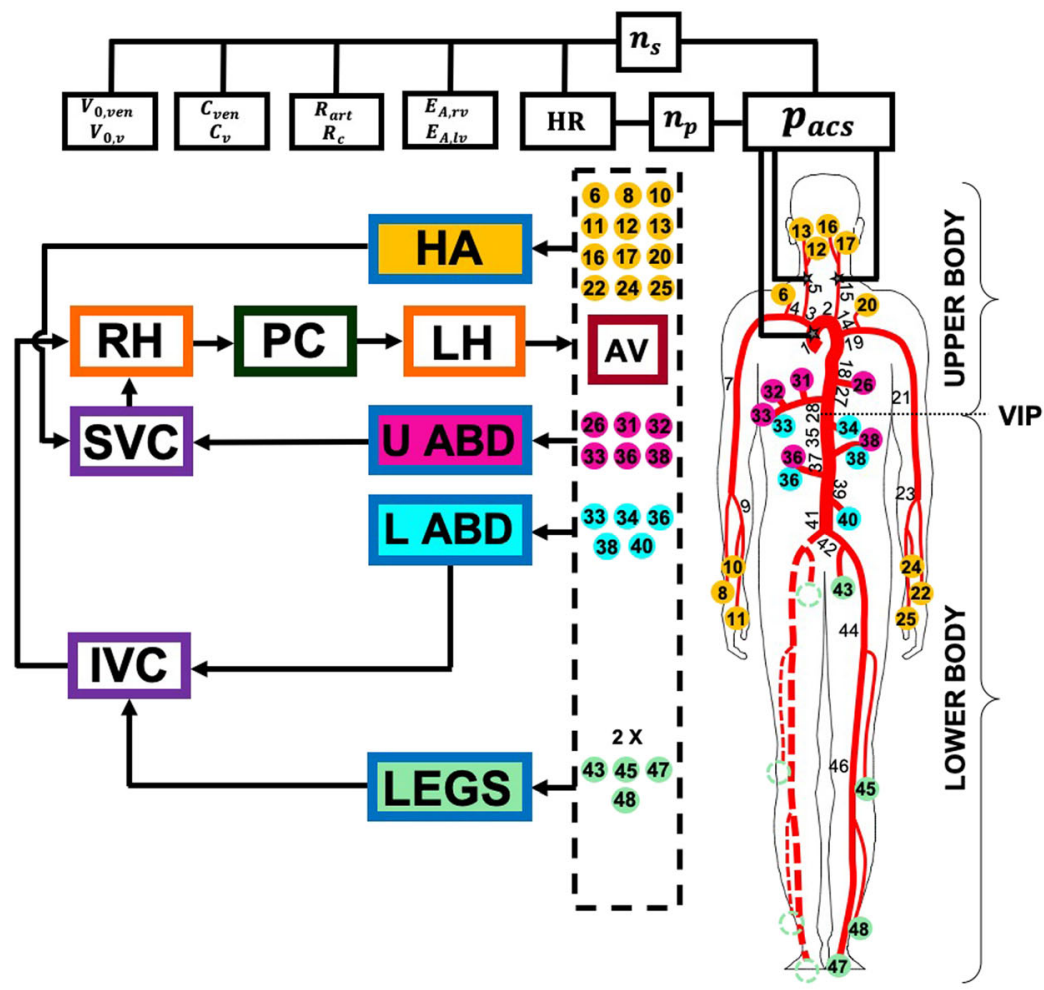

OD SCHEME FOR THE GROUPS: HA, U ABD (CONVERGING INTO SVC), L ABD, LEGS (CONVERGING INTO IVC)

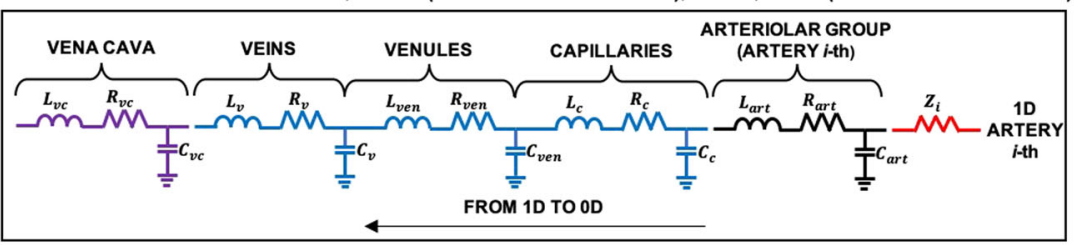

OD SCHEME FOR THE GROUPS: RH (RA \& TV, RV \& PV), PC (P ART, P VEN), LH (LA \& MV, LV \& AV)

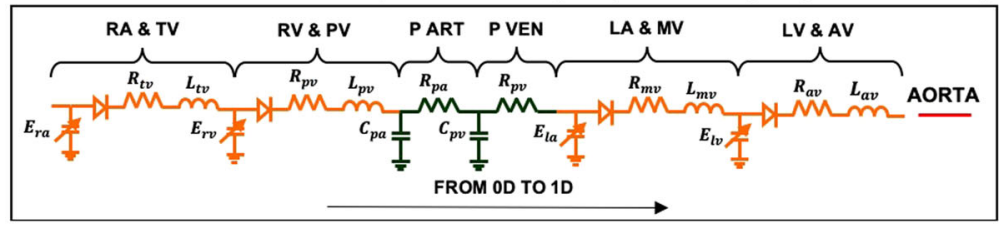

Fig. 5 Scheme of the multiscale model. The sketch includes the arterial tree (right), the structure of the 0D compartments (left), the arteriolar 0D-1D interface (dashed box), and the baroreceptor mechanisms (top). Details of the OD compartments are depicted in the bottom panels. According to VIP, the model is divided into upper body (RH right heart, PC pulmonary circulation, LH left heart, U ABD and HA upper body arteries and arterioles; upper abdomen, head and arms venous return, SVC superior vena cava,) and lower body (L ABD and LEGS lower body arteries and arterioles; lower abdomen and legs venous return, IVC inferior vena cava). Modeling parameters and variables are defined in the Supplementary Information, see Supplementary Table 1 for the legend.

parabolic flight data, which is more informative since blood shift is extinguished in few hours. Within the subset of spaceflight data priority has been given to long-term and recent space missions. By analyzing the selected literature we based the model setting and validation primarily on long-term data having routine and involuntary countermeasures, as well as countermeasures not expressly declared. We did not directly use (for setting or validation) studies adopting particular ad hoc countermeasures, but accounted for them only in order to better contextualize and qualitatively compare our results. Despite the great caution in collecting and managing spaceflight information, it should be kept in mind that: (i) spaceflight literature data often reports variations with respect to the $1 \mathrm{G}$ upright condition, while the present model has been derived in $1 \mathrm{G}$ supine condition for clinical interest. For this reason, when possible, further estimates were done to convert $1 \mathrm{G}$ upright condition measures to $1 \mathrm{G}$ supine conditions; (ii) older data are usually without any countermeasure program while the more recent ones adopt a wide variety of different countermeasures. Although more recent studies were preferred, we took advantage of older measurements (usually without countermeasures) to properly weight the possible effects of countermeasures; (iii) there are overall few long-term missions and data are not always in agreement (usually because of a different type of experimental setting and reference position). This last aspect made the spaceflight setting even more challenging, since a significant part of the analyzed data has been considered not sufficiently reliable for present purposes.

The main cardiovascular changes due to weightlessness introduced into the model were related to blood shift, blood volume reduction, leg venous compliance, cardiac function, arterial resistance, and baroreflex response. Table 5 summarizes the mechanisms considered, the resulting cardiovascular changes and details, where necessary, about their modeling implementation. For blood shift, once VIP was located, blood volume 
Table 5. Summary of the macroscopic mechanisms considered, the adopted cardiovascular changes with respect to the $1 \mathrm{G}$ supine condition and details of the corresponding modeling implementation.

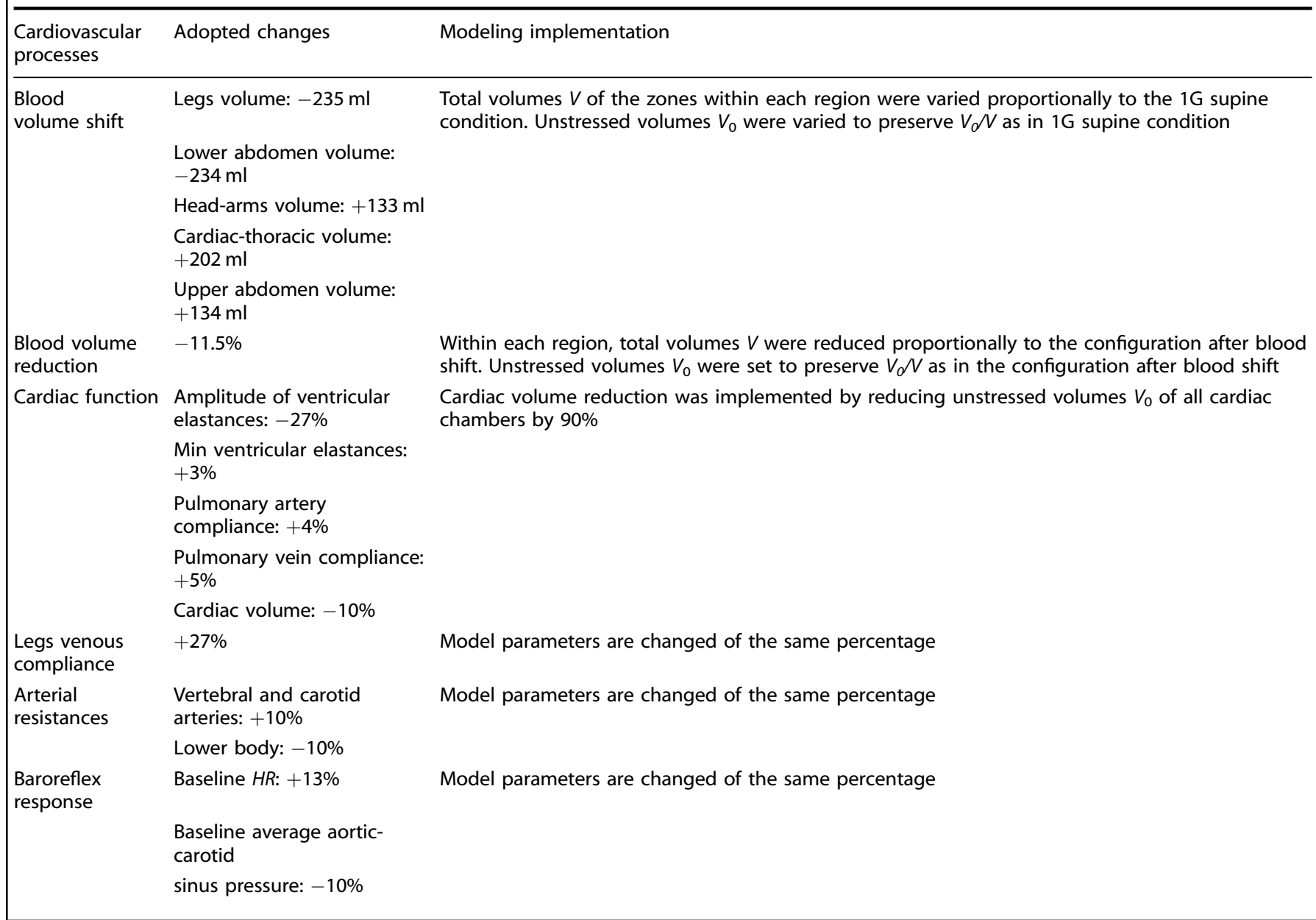

was shifted from lower to upper body using parabolic flight data and based on both the vessel distensibility and the distance from VIP. Total blood volume was then reduced by $11.5 \%$, in accordance with long-term spaceflight data. To account for the contractility and volume reductions ventricular elastances were modified and cardiac volume was decreased. Leg venous compliance was increased by $27 \%$, while arterial resistances were changed according to the body region $(+10 \%$ for vertebral and carotid arteries; $-10 \%$ for the lower body), as observed after long exposure to weightlessness. For baroreflex mechanisms only baseline values were altered as we were interested in long-term spaceflight in steady-state conditions: $H R$ was increased by $13 \%$ and average aortic-carotid sinus pressure was decreased by $10 \%$. An extended description of the hemodynamic considerations and the resulting estimated variations for each cardiovascular mechanism is detailed in the Supplementary Information, together with the corresponding bibliographic references supporting the adopted changes.

\section{Limitations}

A limiting aspect of the present model is that it does not directly control interstitial fluid shift, since we can only act directly on blood volume and blood shift. Nevertheless, interstitial fluid shift and other long-term mechanisms - such as muscle atrophy and blood volume reductionwere intrinsically accounted for in the setting of the long-term spaceflight configuration by modifying the total and unstressed volumes of all cardiovascular compartments. In this regard, effects-such as net diuresis and fluid re-equilibration, fluid shift from intravascular to interstitial space, and the possibility of reduced thirst-were not individually accounted for, but overall were included in the blood volume reduction mechanism.
The model currently does not explicitly include a gravity force term since its effects were modeled based on the available microgravity literature. A gravity force term can be added to the model equations and adjusted to appropriately reproduce lunar, Martian, or other gravity conditions. At present this use would be purely predictive but very helpful in planning future interplanetary missions and extraterrestrial human occupancy.

\section{Reporting summary}

Further information on experimental design is available in the Nature Research Reporting Summary linked to this article.

\section{DATA AVAILABILITY}

The datasets generated and analyzed during the current study are available from the corresponding author on reasonable request.

Received: 17 January 2020; Accepted: 10 August 2020; Published online: 01 October 2020

\section{REFERENCES}

1. Norsk, P., Asmar, A., Damgaard, M. \& Christensen, N. J. Fluid shifts, vasodilatation and ambulatory blood pressure reduction during long duration spaceflight. J. Physiol. 593, 573-584 (2015).

2. Buckey, J. C. Jr. Space Physiology. (Oxford University Press, 2006).

3. Clément, G. Fundamentals of Space Medicine. (Springer, 2011). 
4. Arbeille, P. et al. Adaptation of the left heart, cerebral and femoral arteries, and jugular and femoral veins during short- and long-term head-down tilt and spaceflight. Eur. J. Appl. Physiol. 86, 157-168 (2001).

5. Grigoriev, A. I., Kotovskaya, A. R. \& Fomina, G. A. The human cardiovascular system during space flight. Acta Astronaut. 68, 1495-1500 (2011).

6. Hughson, R. L. et al. Cardiovascular regulation during long-duration spaceflights to the International Space Station. J. Appl. Physiol. 112, 719-727 (2012).

7. Aubert, A. E., Beckers, F. \& Verheyden, B. Cardiovascular function and basics of physiology in microgravity. Acta Cardiol. 60, 129-151 (2005).

8. Planel, H. Space and Life: An Introduction to Space Biology and Medicine. (CRC Press, 2004).

9. Shen, M. \& Frishman, W. H. Effects of spaceflight on cardiovascular physiology and health. Cardiol. Rev. 27, 122-126 (2019).

10. Zhu, H., Wang, H. \& Liu, Z. Effects of real and simulated weightlessness on the cardiac and peripheral vascular function on humans: a review. Int. J. Occup. Med. Environ. Health 28, 793-802 (2015).

11. Hughson, R. L., Helm, A. \& Durante, M. Heart in space: effects of the extraterrestrial environment on the cardiovascular system. Nat. Rev. Cardiol. 15, 167-180 (2018)

12. Norsk, P. Adaptation of the cardiovascular system to weightlessness: surprises, paradoxes and implications for deep space missions. Acta Physiol. 228, e13434 (2020).

13. Gunga, H. C., Weller von Ahlefeld, V., AppellCoriolano, H. J., Werner, A. \& Hoffmann, U. Cardiovascular system, red blood cells, and oxygen transport in microgravity. (Springer, 2016).

14. Gerber, B., Singh, J.-L., Zhangb, Y. \& Liou, W. A computer simulation of short-term adaptations of cardiovascular hemodynamics in microgravity. Comput. Biol. Med. 102, 86-94 (2018).

15. DiazArtiles, A., Heldt, T. \& Young, L. R. Computational model of cardiovascular response to centrifugation and lower body cycling exercise. J. Appl. Physiol. 127, 1453-1468 (2019).

16. Keith, S. M., Batzel, J. J. \& Montani, J. P. Space physiology IV: mathematical modeling of the cardiovascular system in space exploration. Eur. J. Appl. Physiol. 113, 1919-1937 (2013)

17. Lakin, W. D., Stevens, S. A. \& Penar, P. L. Modeling intracranial pressures in microgravity: the influence of the blood-brain barrier. Aviat. Space Environ. Med. 78, 932-936 (2007)

18. Kim, C. S., Kirist, C., Kwak, D. \& David, T. Numerical models of human circulatory system under altered gravity: brain circulation. AIAA Paper 6794-6806. https://doi.org/10.2514/6.2004-1092 (2004).

19. Mynard, J. P. \& Smolich, J. J. One-dimensional haemodynamic modeling and wave dynamics in the entire adult circulation. Ann. Biomed. Eng. 43, 1443-1460 (2015).

20. Blanco, P. J. \& Feijóo, R. A. A dimensionally-heterogeneous closed-loop model for the cardiovascular system and its applications. Med. Eng. Phys. 35, 652-667 (2013).

21. Reymond, P., Bohraus, Y., Perren, F., Lazeyras, F. \& Stergiopulos, N. Validation of a patient-specific one-dimensional model of the systemic arterial tree. Am. J. Physiol. Heart Circ. 301, 1173-1183 (2011).

22. Aboelkassem, Y. \& Virag, Z. A hybrid Windkessel-Womersley model for blood flow in arteries. J. Theor. Biol. 462, 499-513 (2019).

23. Ryu, J., Hu, X. \& Shadden, S. C. A coupled lumped-parameter and distributed network model for cerebral pulse-wave hemodynamics. J. Biomech. Eng. 137, 101009 (2015)

24. Guala, A., Camporeale, C. \& Ridolfi, L. Compensatory effect between aortic stiffening and remodelling during ageing. PLOS ONE 10, e0139211 (2015).

25. Guala, A., Scalseggi, M. \& Ridolfi, L. Coronary fluid mechanics in an ageing cardiovascular system. Meccanica 52, 503-514 (2017).

26. Mirramezani, M., Diamond, S. L., Litt, H. I. \& Shadden, S. C. Reduced order models for transstenotic pressure drop in the coronary arteries. J. Biomech. Eng. 141, 031005 (2019).

27. Mynard, J. P. \& Smolich, J. J. Influence of anatomical dominance and hypertension on coronary conduit arterial and microcirculatory flow patterns: a multiscale modeling study. Am. J. Physiol. Heart Circ. Physiol. 311, H11-23 (2016).

28. Scarsoglio, S., Gallo, C. \& Ridolfi, L. Effects of atrial fibrillation on the arterial fluid dynamics: a modelling perspective. Meccanica 53, 3251-3267 (2018).

29. Scarsoglio, S., Gallo, C., Saglietto, A., Ridolfi, L. \& Anselmino, M. Impaired coronary blood flow at higher heart rates during atrial fibrillation: Investigation via multiscale modelling. Comp. Meth. Progr. Biomed. 175, 95-102 (2019).

30. Saglietto, A., Scarsoglio, S., Ridolfi, L., Gaita, F. \& Anselmino, M. Higher ventricular rate during atrial fibrillation relates to increased cerebral hypoperfusions and hypertensive events. Sci. Rep. 9, 3779 (2019).

31. Gallo, C., Ridolfi, L. \& Scarsoglio, S. A closed-loop multiscale model of the cardiovascular system: application to heart pacing and open-loop response, IFMBE Proc. 76, 577-585 (2020).
32. Guala, A., Camporeale, C., Tosello, F., Canuto, C. \& Ridolfi, L. Modelling and subject-specific validation of the heart-arterial tree system. Ann. Biomed. Eng. $\mathbf{4 3}$ 222-237 (2014)

33. Tosello, F. et al. Central pressure appraisal: clinical validation of a subject-specific mathematical model. PLOS ONE 11, e0151523 (2016).

34. Guala, A., Leone, D., Milan, A. \& Ridolfi, L. In silico analysis of the anti-hypertensive drugs impact on myocardial oxygen balance. Biomech. Model. Mechanobiol. 16, 1035-1047 (2017).

35. Liang, F., Takagi, S., Himeno, R. \& Liu, H. Multi-scale modeling of the human cardiovascular system with applications to aortic valvular and arterial stenoses. Med. Biol. Eng. Comp. 47, 743-755 (2009).

36. Herault, S. et al. Cardiac, arterial and venous adaptation to weightlessness during 6-month MIR space flights with and without thigh cuffs (bracelets). Eur. J. Appl. Physiol. 81, 384-390 (2000).

37. Martin, D. S., South, D. A., Wood, M. L., Bungo, M. \& Fritsch-Yelle, J. M. Comparison of echocardiographic measurements before and after short and long duration spaceflight, NASA Technical Reports Server (NTRS) 20100029745 (2000).

38. Martin, D. S., South, D. A., Wood, M. L., Bungo, M. \& Meck, J. V. Comparison of echocardiographic changes after short- and long-duration spaceflight. Aviat Space Environ. Med. 73, 532-536 (2002).

39. Levine, B. D., Zuckerman, J. H. \& Pawelczyk, J. A. Cardiac atrophy after bed-rest deconditioning. Circulation 96, 517-525 (1997).

40. Spaak, J., Montmerle, S., Sundblad, P. \& Linnarsson, D. Long-term bed restinduced reductions in stroke volume during rest and exercise: cardiac dysfunction vs. volume depletion. J. Appl. Physiol. 98, 648-654 (2005).

41. Perhonen, M. A., Zuckerman, J. H. \& Levine, B. D. Deterioration of left ventricular chamber performance after bed rest. Circulation 103, 1851-1857 (2001).

42. Marshall-Goebel, K. et al. Assessment of jugular venous blood flow stasis and thrombosis during spaceflight. JAMA Netw. Open. 2, e1915011 (2019).

43. Verheyden, B., Liu, J., Beckers, F. \& Aubert, A. E. Adaptation of heart rate and blood pressure to short and long duration space missions. Respir. Physiol. Neurobiol. 169S, S13-S16 (2009).

44. Verheyden, B., Liu, J., Beckers, F. \& Aubert, A. E. Operational point of neural cardiovascular regulation in humans up to 6 months in space. J. Appl. Physiol. 108, 646-654 (2010)

45. Ade, C. J., Broxterman, R. M., Moore, A. D. \& Barstow, T. J. Decreases in maximal oxygen uptake following long-duration spaceflight: Role of convective and diffusive $\mathrm{O}_{2}$ transport mechanisms. J. Appl. Physiol. 122, 968-975 (2017).

46. Buckey, J. C. et al. Central venous pressure in space. N. Engl. J. Med. 328 1853-1854 (1993).

47. Buckey, J. C. et al. Central venous pressure in space. J. Appl. Physiol. 81, 19-25 (1996).

48. Videbaek, R. \& Norsk, P. Atrial distension in humans during microgravity induced by parabolic flights. J. Appl. Physiol. 83, 1862-1866 (1997).

49. Foldager, N. et al. Central venous pressure in humans during microgravity. J. Appl. Physiol. 1985, 408-412 (1996).

50. Zhang, L. F. \& Hargens, A. R. Spaceflight-induced intracranial hypertension and visual impairment: pathophysiology and countermeasures. Physiol. Rev. 98, 59-87 (2018).

51. Caro, C. G., Pedley, T. J., Schroter, R. C. \& Seed, W. A. The mechanics of the circulation 2nd edn, (Cambridge University Press, New York, 2012).

52. Westerhof, N., Stergiopulos, N. \& Noble, M. I. M. Snapshots of Hemodynamics 2nd ed. (Springer, Berlin, 2010).

53. Fomina, G. A., Kotovskaya, A. R. \& Temnova, E. V. Dynamics of human cardiovascular responses in different periods of longterm exposure to weightlessness. Hum. Physiol. 38, 715-720 (2012).

54. Miyachi, M. et al. Greater age-related reductions in central arterial compliance in resistance-trained men. Hypertension 41, 130-135 (2003).

55. Bertovic, D. A. et al. Muscular strength training is associated with low arterial compliance and high pulse pressure. Hypertension 33, 1385-1391 (1999).

56. Cortez-Cooper, M. Y. et al. Effects of high intensity resistance training on arterial stiffness and wave reflection in women. Am. J. Hypertens. 18, 930-934 (2005).

57. Koeppen, B. \& Stanton, B. Berne \& Levy Physiology 6th edn, (Elsevier, 2009).

58. Guala, A. et al. Multiscale mathematical modeling vs. the generalized transfer function approach for aortic pressure estimation: a comparison with invasive data. Hypertens. Res. 42, 690-698 (2019).

\section{ACKNOWLEDGEMENTS}

The authors would like to thank Claudio Canuto for the precious help with the numerical implementation of the model, Matteo Anselmino for the fruitful discussion of the results, and Mark Miller for his valuable contributions to the editing of the manuscript. 


\section{AUTHOR CONTRIBUTIONS}

S.S. and L.R. conceived and designed the experiments. C.G. performed the experiments. S.S. and L.R. analyzed the data. All authors contributed reagents/ materials/analysis tools. S.S. wrote the paper. All authors reviewed and approved the final version of the manuscript.

\section{COMPETING INTERESTS}

The authors declare no competing interests.

\section{ADDITIONAL INFORMATION}

Supplementary information is available for this paper at https://doi.org/10.1038/ s41526-020-00117-5.

Correspondence and requests for materials should be addressed to S.S.

Reprints and permission information is available at http://www.nature.com/ reprints
Publisher's note Springer Nature remains neutral with regard to jurisdictional claims in published maps and institutional affiliations.

Open Access This article is licensed under a Creative Commons adaptation, distribution and reproduction in any medium or format, as long as you give appropriate credit to the original author(s) and the source, provide a link to the Creative Commons license, and indicate if changes were made. The images or other third party material in this article are included in the article's Creative Commons license, unless indicated otherwise in a credit line to the material. If material is not included in the article's Creative Commons license and your intended use is not permitted by statutory regulation or exceeds the permitted use, you will need to obtain permission directly from the copyright holder. To view a copy of this license, visit http://creativecommons. org/licenses/by/4.0/.

(c) The Author(s) 2020 